\title{
Conductivity of disordered electrons: Mean-field approximation containing vertex corrections
}

\author{
V. Janis ${ }^{1}$ and D. Vollhardt ${ }^{2}$ \\ ${ }^{1}$ Institute of Physics, Academy of Sciences of the Czech Republic, Na Slovance 2, CZ-18221 Praha 8, Czech Republic \\ ${ }^{2}$ Theoretische Physik III, Elektronische Korrelationen und Magnetismus, Institut für Physik, Universität Augsburg, \\ D-86135 Augsburg, Germany
}

(Received 25 August 2000; published 12 March 2001)

\begin{abstract}
The electrical dc-conductivity of disordered, noninteracting electrons is calculated in the asymptotic limit of high lattice dimensions $d \rightarrow \infty$. To go beyond the lowest-order contribution in the expansion parameter $1 / d$ of the single bubble diagram, vertex corrections are calculated from an asymptotic expression for the two-particle vertex. A mean-field approximation for the dc conductivity containing the leading high-dimensional vertex corrections is proposed which is free of spurious nonanalyticities, i.e., the conductivity is non-negative and shows no unphysical behavior in $d \geqslant 3$.
\end{abstract}

DOI: 10.1103/PhysRevB.63.125112

PACS number(s): 72.80.Ng, 71.23.An

Electronic problems with interactions or disorder can almost never be solved exactly, except for special limits. The resolvent functions of an exact solution fulfill conservation laws and have the correct analytic (Herglotz) properties, i.e., do not contain spurious poles. This is generally not true for approximate, e.g., perturbative, solutions. Only global, selfconsistent approximations have a chance to be free of unphysical behavior and to yield the desired analyticity of a solution for all input parameters.

For noninteracting tight-binding electrons in a random potential the first self-consistent solution with the correct analytic properties was the "coherent potential approximation", (CPA). ${ }^{1,2}$ The Herglotz analyticity of the CPA equations was proved explicitly by Müller-Hartmann. ${ }^{3}$ Only later the CPA was found to be the exact solution of the Anderson disorder model in two particular limits. First, the CPA with a semicircular disorder distribution was shown to correspond to the exact solution of an $n$-orbital model in the limit $n=\infty .4$ Then, after the limit of high spatial dimensions $d$ for fermionic lattice models had been introduced, ${ }^{5}$ it was found that the CPA represents the exact solution of the Anderson disorder model in $d=\infty$ for arbitrary disorder distributions. ${ }^{6,7}$ Since then the limit $d \rightarrow \infty$ has served as a useful tool for deriving self-consistent, fully dynamical approximation schemes for interacting lattice electron systems, ${ }^{8}$ referred to as dynamical mean-field theory (DMFT).

By calculating a physical quantity in $d=\infty$ one obtains a particular mean-field value. The situation becomes subtle if the value obtained in this limit is zero. This is, for example, the case for nonlocal quantities such as the off-diagonal propagator $G_{i j}, i \neq j$. They depend on the distance between two or more different lattice sites (i.e., their Fourier transform is wave-vector dependent) and are thus necessarily proportional to some power of $1 / d$, e.g., $G_{i j} \sim O(1 / \sqrt{d})$, for nearest-neighbor sites $i, j$. However, that does not imply that these quantities can be neglected in $d=\infty$. They may contribute, since they appear in lattice sums where the summation over the $d \rightarrow \infty$ many sites compensates their $1 / d$ smallness. To include these quantities properly, one has to calculate their asymptotic behavior in the limit $d \rightarrow \infty$, thus going beyond the strict $d=\infty$ limit. The necessity to go be- yond $d=\infty$ is also evident from the fact that higher-order nonlocal Green functions are related to lower-order local Green functions by functional derivatives via generalized Ward identities, reflecting conservation laws. It was recently shown by one of us ${ }^{9}$ and by Hettler et al. ${ }^{10}$ that, when only the leading asymptotic contributions to one- and two-particle Green functions are taken into account, the Ward identities are not fulfilled in $d=\infty$. One has to go beyond the leading order at the lower particle level, or introduce anomalous functions, to restore conservation laws. ${ }^{9}$ This shows that the definition of two- and higher-order Green functions is ambiguous in the limit $d=\infty$.

A particularly important example of a quantity whose mean-field value in $d=\infty$ vanishes, is the electrical conductivity $\sigma$. It is defined from a Kubo formula with the currentcurrent correlation function. In the limit $d \rightarrow \infty$ the optical dc conductivity is given by a single bubble diagram, with $\sigma$ $\sim O(1 / d) .{ }^{11}$ In a formal $1 / d$ expansion this result would be the first nonvanishing contribution to $\sigma$. It is useful to consider this nonvanishing asymptotic result as a "mean-field value" of the conductivity. ${ }^{12}$ Likewise one may define a mean-field value of any physical quantity through its leading nonvanishing asymptotic result in the limit $d \rightarrow \infty$.

A mean-field result for the dc conductivity $\sigma \sim O(1 / d)$ defined in this way does not contain vertex corrections. Hence it does not include the physics of backscatterings. However, in random systems vertex corrections are known to be extremely important since they are responsible for Anderson localization at zero temperature in sufficiently low dimensions $(d=1,2)$ or for sufficiently strong disorder in 3 $\leqslant d<\infty$. At least from a diagrammatic point of view it is not yet fully understood how the conductivity $\sigma$ approaches zero at the localization transition. ${ }^{13,14}$ Clearly one has to go beyond the mean-field single-site diagrams to incorporate localization effects.

Most recently, Jarrell and Krishnamurthy ${ }^{15}$ introduced systematic nonlocal corrections to the CPA on the oneparticle level using the dynamical cluster approximation to obtain results compatible with Herglotz analyticity, i.e., a non-negative density of states. Here we choose another route 
to go beyond the mean-field limit and calculate nonlocal corrections to the CPA two-particle irreducible vertex function. The aim of our paper is to employ the limit of high lattice dimensions to improve upon the mean-field conductivity $\sigma$ $\sim O(1 / d)$ by including vertex corrections. We follow the proposal of Ref. 9 where the high-dimensional asymptotics of the full vertex function with leading vertex corrections to the electrical conductivity was derived. Our main result in this paper is a mean-field expression for the electrical conductivity which includes leading asymptotic vertex corrections in $d \rightarrow \infty$ while remaining non-negative in $d \geqslant 3$.

The vertex function from Ref. 9 contains the leading $1 / d$ asymptotics of all two-particle quantities. Employing the Kubo formula for the electrical conductivity with the twoparticle vertex we may derive the leading asymptotics for the conductivity and its vertex corrections. However, approximations of the full vertex function in the Kubo formula can, in principle, lead to unphysical results. Indeed, the vertex corrections to the single-bubble term may have a negative sign and hence positivity of the conductivity cannot be warranted. Clearly, a meaningful and consistent approximation for the conductivity must never become negative. To obtain such an approximation we represent the full vertex function by means of an irreducible vertex and a Bethe-Salpeter equation in the electron-hole channel. To produce the leading contribution to the vertex corrections in $d \rightarrow \infty$, the irreducible function must be evaluated with its leading nonlocal contribution. By simplifying the Bethe-Salpeter equation in high spatial dimensions we then obtain a closed, mean-field expression for the conductivity with vertex corrections.

In the following we consider the Anderson disorder Hamiltonian

$$
H=-\frac{t^{*}}{\sqrt{Z}} \sum_{\langle i j\rangle} c_{i}^{\dagger} c_{j}+\sum_{i} V_{i} c_{i}^{\dagger} c_{i},
$$

to describe the effects of randomness. Here $t^{*}$ is the hopping matrix element between nearest neighbors, scaled in such a way as to produce a meaningful limit $d \rightarrow \infty$, with $Z$ as the coordination number of the lattice. ${ }^{5}$ The local, static potential $V_{i}$ is a random variable with site-independent distribution function. The conductivity of a quenched random system without interparticle interactions is described by averaged one- and two-particle Green functions (resolvents) $G_{i j}(z)$ $=\left\langle[z \hat{1}-\hat{t}-\hat{V}]_{i j}\right\rangle_{\mathrm{av}}$ and $G_{i j, k l}^{(2)}\left(z_{1}, z_{2}\right)=\left\langle\left[z_{1} \hat{1}-\hat{t}-\hat{V}\right]_{i j}^{-1}\left[z_{2} \hat{1}\right.\right.$ $\left.-\hat{t}-\hat{V}]_{k l}^{-1}\right\rangle_{\mathrm{av}}$, respectively. It is our first goal to determine these functions in the asymptotic limit $d \rightarrow \infty$.

It is straightforward to derive the $d \rightarrow \infty$ limit of the selfenergy which carries the information about how the randomness influences the motion of a single electron. The selfenergy becomes local and can be obtained from the singlesite equation

$$
\left\langle\frac{1}{1+\left(\Sigma(z)-V_{i}\right) G(z)}\right\rangle_{\text {av }}=1 .
$$

This is precisely the well-known CPA equation for the self-energy. ${ }^{1}$ Here the local (diagonal) one-particle propaga- tor is denoted by $G(z)=N^{-1} \Sigma_{\mathbf{k}} G(\mathbf{k}, z)=\int d \rho(\epsilon)[z-\Sigma(z)$ $-\epsilon]^{-1}$ where $\rho$ is the density of states. It is less evident how to derive expressions for averaged two-particle functions which are consistent with the local self-energy, since we have to work explicitly with nonlocal quantities. At the twoparticle level one has to keep two separate lattice points to derive the leading asymptotics for large $d .{ }^{9}$ It is more convenient and practical to work with an averaged cumulant, or better with a vertex $\Gamma$ defined in momentum space as

$$
\begin{aligned}
\Gamma\left(\mathbf{k}_{1}, z_{1}, \mathbf{k}_{2}, z_{2} ; \mathbf{q}\right)= & G^{-1}\left(\mathbf{k}_{1}, z_{1}\right) G^{-1}\left(\mathbf{k}_{2}, z_{2}\right) \\
& \times\left[G^{(2)}\left(\mathbf{k}_{1}, z_{1}, \mathbf{k}_{2}, z_{2} ; \mathbf{q}\right)\right. \\
& \left.-\delta(\mathbf{q}) G\left(\mathbf{k}_{1}, z_{1}\right) G\left(\mathbf{k}_{2}, z_{2}\right)\right] \\
& \times G^{-1}\left(\mathbf{k}_{1}+\mathbf{q}, z_{1}\right) G^{-1}\left(\mathbf{k}_{2}+\mathbf{q}, z_{2}\right) .
\end{aligned}
$$

Strictly in $d=\infty$ the two-particle vertex function $\Gamma$ is local, since the one-particle propagators are local. For convenience we denote the local vertex obtained in this limit as $\gamma:=\lim _{d \rightarrow \infty} \Gamma$; it can be obtained from a local Bethe-Salpeter equation, and is given by $\gamma\left(z_{1}, z_{2}\right)=\Lambda\left(z_{1}, z_{2}\right) /[1$ $\left.-\Lambda\left(z_{1}, z_{2}\right) G\left(z_{1}\right) G\left(z_{2}\right)\right]$, where

$$
\begin{aligned}
\Lambda\left(z_{1}, z_{2}\right)= & \frac{\delta \Sigma\left(z_{1}\right)}{\delta G\left(z_{2}\right)} \\
= & \frac{1}{G\left(z_{1}\right) G\left(z_{2}\right)} \\
& \times\left[1-\left\langle\frac{1}{1+\left(\Sigma(z)-V_{i}\right) G\left(z_{1}\right)}\right.\right. \\
& \left.\left.\times \frac{1}{1+\left(\Sigma(z)-V_{i}\right) G\left(z_{2}\right)}\right\rangle_{\text {av }}^{-1}\right]
\end{aligned}
$$

is the two-particle irreducible vertex in CPA. In the following we sketch the derivation of the asymptotic form of the vertex $\Gamma$ in $d \rightarrow \infty$, exact up to order $1 / d$, for the Anderson model of disordered electrons (1) along the lines explained in Ref. 9. For details we refer to Ref. 16, where it is shown that this asymptotic form of $\Gamma$ is obtained as the first iteration of the parquet equations for nonlocal vertices.

In high dimensions, taking into account corrections to order $1 / d$, two lattice sites $i \neq j$ can be connected by maximally two one-particle propagators (i.e., $G_{i j}, G_{j i}$ ). Hence, in this limit two-particle functions only contribute if they carry at most two distinct lattice sites. Since there are three different ways to connect two two-particle functions by two oneparticle propagators the exact asymptotic form of the vertex $\Gamma$ up to order $1 / d$ can be represented as a sum of three contributions, i.e., solutions of Bethe-Salpeter equations in three inequivalent channels. These channels are distinguished by different types of two-particle irreducibility: the electron-hole, electron-electron (hole-hole), and vertical channel, respectively. ${ }^{9}$ The latter corresponds to the interaction channel (' $U$-channel") of Ref. 9 and represents non- 

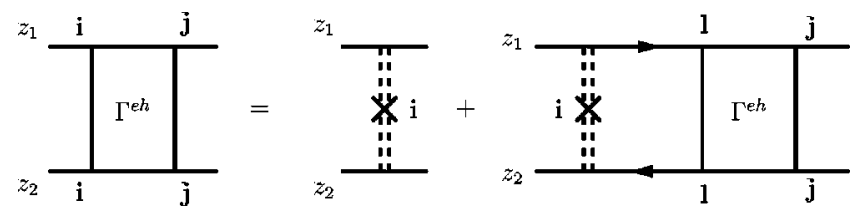

$\mathbf{a}$
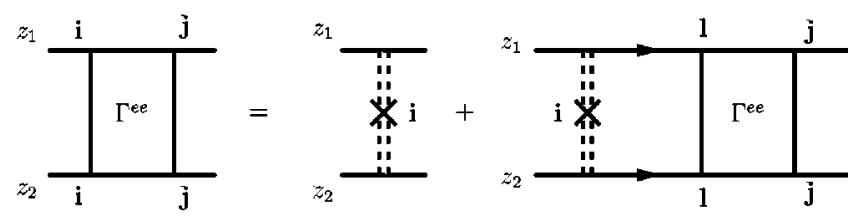

b

FIG. 1. Diagrammatic representation of the Bethe-Salpeter equations (5a) and (5b) for the two-particle vertex functions defined from (a) the electron-hole $\left(\Gamma^{e h}\right)$ and (b) the electron-electron $\left(\Gamma^{e e}\right)$ channels. Oriented solid lines represent nonlocal one-electron propagators; the double-dashed line represents the local vertex $\gamma$ obtained in the limit $d=\infty$. The lattice index $\mathbf{I}$ is summed over.

local self-scattering corrections. The three contributions $\Gamma^{e h}$, $\Gamma^{e e}$, and $\Gamma^{v}$ are represented diagrammatically (in lattice space) in Fig. 1 and Fig. 2. The vertices $\Gamma^{e h}$ and $\Gamma^{e e}$ obtained from the electron-hole and electron-electron channel, respectively, are given by

$$
\begin{aligned}
\Gamma_{i j}^{e h}\left(z_{1}, z_{2}\right)= & \gamma\left(z_{1}, z_{2}\right) \delta_{i, j} \\
& +\gamma\left(z_{1}, z_{2}\right) \sum_{l \neq i} G_{i l}\left(z_{1}\right) G_{l i}\left(z_{2}\right) \Gamma_{l j}^{e h}\left(z_{1}, z_{2}\right) \\
\Gamma_{i j}^{e e}\left(z_{1}, z_{2}\right)= & \gamma\left(z_{1}, z_{2}\right) \delta_{i, j} \\
& +\gamma\left(z_{1}, z_{2}\right) \sum_{l \neq i} G_{i l}\left(z_{1}\right) G_{i l}\left(z_{2}\right) \Gamma_{l j}^{e e}\left(z_{1}, z_{2}\right)
\end{aligned}
$$

The Bethe-Salpeter equation for the sum of the reducible diagrams in the vertical channel is slightly more complicated (Fig. 2):

$$
\begin{aligned}
\Gamma_{i j}^{v}\left(z_{1}, z_{2}\right)= & \gamma\left(z_{1}, z_{2}\right) \delta_{i, j} \\
& +\gamma\left(z_{1}, z_{1}\right) \sum_{l \neq i} G_{i l}\left(z_{1}\right) G_{l i}\left(z_{1}\right) \Gamma_{l j}^{v}\left(z_{1}, z_{2}\right) \\
& +\sum_{l \neq j} \Gamma_{i l}^{v}\left(z_{1}, z_{2}\right) G_{l j}\left(z_{2}\right) G_{j l}\left(z_{2}\right) \gamma\left(z_{2}, z_{2}\right) \\
& -\gamma\left(z_{1}, z_{1}\right) \sum_{l \neq i} G_{i l}\left(z_{1}\right) \\
& \times G_{l i}\left(z_{1}\right) \Gamma_{l m}^{v}\left(z_{1}, z_{2}\right) G_{m j}\left(z_{2}\right) G_{j m}\left(z_{2}\right) \gamma\left(z_{2}, z_{2}\right) .
\end{aligned}
$$

The last term in the equation for $\Gamma^{v}$ carries a minus sign to compensate for diagrams included in the two preceding terms. Unlike the case of interacting electrons, the asymptotic Bethe-Salpeter equations for noninteracting disordered electrons can be solved explicitly, since they are algebraic in momentum space. Instead of using the local vertex $\gamma$ and the non-local part of the one-particle propagators in the Bethe-Salpter equations one may equally work with the irreducible vertex $\Lambda$ from Eq. (4) and bubbles of oneparticle propagators, defined in momentum space as $\chi^{ \pm}\left(\mathbf{q} ; z_{1}, z_{2}\right)=N^{-1} \sum_{\mathbf{k}} G\left(\mathbf{k}, z_{1}\right) G\left(\mathbf{q} \pm \mathbf{k}, z_{2}\right)$. Using the above definitions the solutions of the Bethe-Salpeter equations in the three two-particle channels take the form (in momentum space):

$$
\begin{gathered}
\Gamma^{e h}\left(\mathbf{q} ; z_{1}, z_{2}\right)=\frac{\Lambda\left(z_{1}, z_{2}\right)}{1-\Lambda\left(z_{1}, z_{2}\right) \chi^{+}\left(\mathbf{q} ; z_{1}, z_{2}\right)} \\
\Gamma^{e e}\left(\mathbf{q} ; z_{1}, z_{2}\right)=\frac{\Lambda\left(z_{1}, z_{2}\right)}{1-\Lambda\left(z_{1}, z_{2}\right) \chi^{-}\left(\mathbf{q} ; z_{1}, z_{2}\right)}, \\
\Gamma^{v}\left(\mathbf{q} ; z_{1}, z_{2}\right)=\gamma\left(z_{1}, z_{2}\right) \prod_{i=1}^{2} \frac{1-\Lambda\left(z_{i}, z_{i}\right) G\left(z_{i}\right) G\left(z_{i}\right)}{\left[1-\Lambda\left(z_{i}, z_{i}\right) \chi^{+}\left(\mathbf{q} ; z_{i}, z_{i}\right)\right]} .
\end{gathered}
$$

The full vertex is a sum of the above three contributions, where the transferred momentum $\mathbf{q}$ has a different meaning in each channel. This is due to the fact that the irreducibility

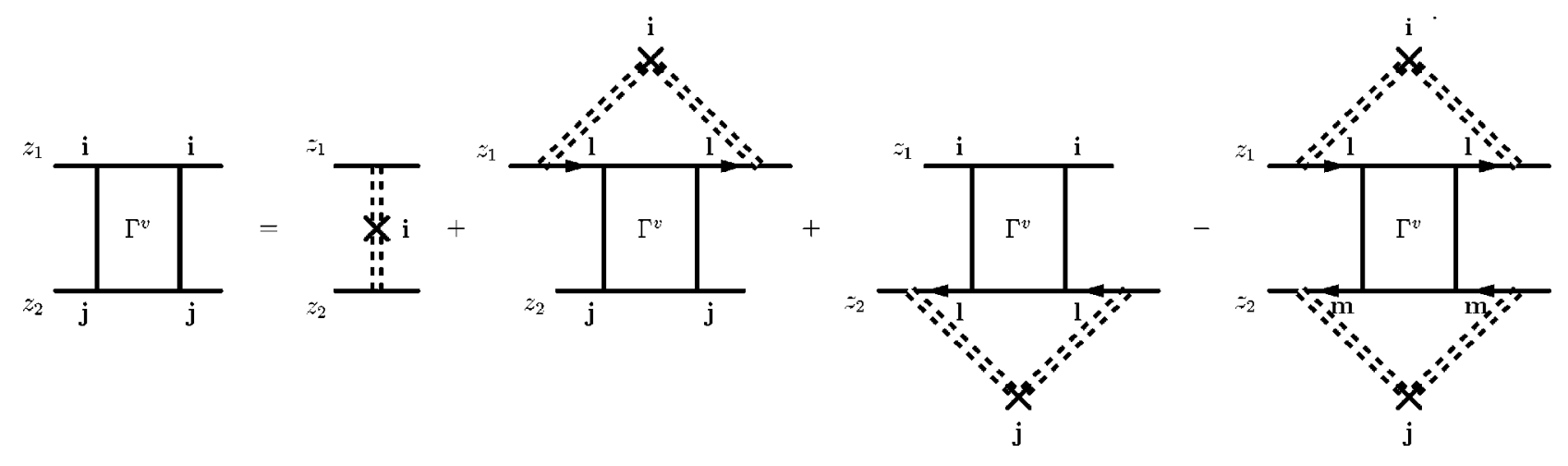

FIG. 2. Diagrammatic representation of the Bethe-Salpeter equation (5c) for the two-particle vertex function $\Gamma^{v}$ in the vertical channel. The lattice indices $\mathbf{l}$ and $\mathbf{m}$ are summed over. 
channels are topologically inequivalent and differ in the momentum that is conserved during multiple scatterings. If the incoming particle and hole carry momenta $\mathbf{k}_{1}$ and $\mathbf{k}_{2}$ then the conserved momentum is $\mathbf{k}_{2}-\mathbf{k}_{1}, \mathbf{k}_{1}+\mathbf{k}_{2}+\mathbf{q}$, and $\mathbf{q}$ for the electron-hole, electron-electron, and vertical channels, respectively. ${ }^{17}$ The momentum $\mathbf{q}$ is the momentum transferred during the scattering on impurities, i.e., the outgoing particle and hole carry momenta $\mathbf{k}_{1}+\mathbf{q}$ and $\mathbf{k}_{2}+\mathbf{q}$, respectively. In order to avoid multiple summation on the same site we must subtract the local vertex from the sum of the channel-dependent vertex functions twice. We then obtain an explicit representation for the two-particle vertex in high dimensions in the notation of Ref. 9

$$
\begin{aligned}
\Gamma\left(\mathbf{k}_{1}, z_{1}, \mathbf{k}_{2}, z_{2} ; \mathbf{q}\right)= & \Gamma^{e h}\left(\mathbf{k}_{2}-\mathbf{k}_{1} ; z_{1}, z_{2}\right)+\Gamma^{e e}\left(\mathbf{k}_{1}+\mathbf{k}_{2}\right. \\
& \left.+\mathbf{q} ; z_{1}, z_{2}\right)+\Gamma^{v}\left(\mathbf{q} ; z_{1}, z_{2}\right)-2 \gamma\left(z_{1}, z_{2}\right) .
\end{aligned}
$$

We note that the CPA vertex function derived in Ref. 18 is given by only the first term in the above equation, i.e., $\Gamma^{e h}$. Hence it does not contain the transferred momentum q needed to incorporate vertex corrections to the conductivity.

The static $(\mathrm{dc})$ electrical conductivity at zero temperature is defined by a Kubo formula with the full vertex as ( $\hbar$ $=1$ )

$$
\begin{aligned}
\operatorname{Re} \sigma_{\alpha \beta}= & \frac{e^{2}}{4 \pi} \frac{1}{N^{2}} \sum_{\mathbf{k}, \mathbf{k}^{\prime}} v_{\alpha}(\mathbf{k}) v_{\beta}\left(\mathbf{k}^{\prime}\right) \sum_{\sigma \tau}(-\sigma \tau) G_{\sigma}(\mathbf{k}) G_{\tau}(\mathbf{k}) \\
& \times\left[\delta\left(\mathbf{k}-\mathbf{k}^{\prime}\right)+\Gamma_{\sigma \tau}\left(\mathbf{k}, \mathbf{k} ; \mathbf{k}^{\prime}-\mathbf{k}\right) G_{\sigma}\left(\mathbf{k}^{\prime}\right) G_{\tau}\left(\mathbf{k}^{\prime}\right)\right]
\end{aligned}
$$

where $\quad \sigma, \tau= \pm 1, \quad \Gamma_{\sigma \tau}\left(\mathbf{k}, \mathbf{k}^{\prime} ; \mathbf{q}\right)=\Gamma\left(\mathbf{k}, E_{F}+i \sigma 0^{+}, \mathbf{k}^{\prime}, E_{F}\right.$ $\left.+i \tau 0^{+} ; \mathbf{q}\right), G_{\sigma}(\mathbf{k})=G\left(\mathbf{k}, E_{F}+i \sigma 0^{+}\right), \quad v_{\alpha}(\mathbf{k})=m^{-1} \partial \epsilon(\mathbf{k}) /$ $\partial k_{\alpha}, \epsilon(\mathbf{k})$ is the dispersion relation and $m$ the mass of the electron.

Equation (8) with the vertex functions (6) contains nontrivial corrections to the one-electron conductivity (the single-bubble diagram). However, Eq. (8) is not appropriate for approximate evaluations in finite dimensions. Namely, the vertex corrections are merely added to the one-particle conductivity such that in an approximation negative contributions may reverse the overall sign, thereby leading to unphysical behavior. To avoid such a situation we will now represent the conductivity in a different, but equivalent, way. We use a Bethe-Salpeter equation in the electron-hole channel expressing the full vertex $\Gamma$ via an irreducible one, $\Lambda^{e h}$. The irreducible vertex $\Lambda^{\text {eh }}$ together with $\chi^{+}$determine the integral kernel of the Bethe-Salpeter equation explicitly and define a matrix multiplication scheme in momentum space. ${ }^{17}$ The integral kernel and the multiplication rule for the electron-hole channel are given by

$$
\left[\Lambda_{\sigma \tau}^{e h} G_{\sigma} G_{\tau}\right]\left(\mathbf{k}, \mathbf{k}^{\prime} ; \mathbf{q}\right)=\Lambda_{\sigma \tau}^{e h}\left(\mathbf{k}, \mathbf{k}^{\prime} ; \mathbf{q}\right) G_{\sigma}(\mathbf{k}+\mathbf{q}) G_{\tau}\left(\mathbf{k}^{\prime}+\mathbf{q}\right),
$$

$$
\begin{aligned}
{[X \bullet Y]\left(\mathbf{k}, \mathbf{k}^{\prime} ; \mathbf{q}\right)=} & \frac{1}{N} \sum_{\mathbf{q}^{\prime}} X\left(\mathbf{k}, \mathbf{k}^{\prime} ; \mathbf{q}^{\prime}\right) \\
& \times Y\left(\mathbf{k}+\mathbf{q}^{\prime}, \mathbf{k}^{\prime}+\mathbf{q}^{\prime} ; \mathbf{q}-\mathbf{q}^{\prime}\right) .
\end{aligned}
$$

A solution of the Bethe-Salpeter equation for the twoparticle vertex $\Lambda^{\text {eh }}$ can formally be written as $\Gamma=\{1$ $\left.-\left[\Lambda^{e h} G G\right] \bullet\right\}^{-1} \Lambda^{e h}$ where the bullet indicates that, upon expansion of $\{\ldots\}^{-1}$, the two-particle functions $\left[\Lambda^{\text {eh }} G G\right]$ are multiplied according to Eq. (9b). Inserting this solution into Eq. (8) we obtain an equivalent representation for the conductivity

$$
\begin{aligned}
\operatorname{Re} \sigma_{\alpha \beta}= & \frac{e^{2}}{4 \pi} \frac{1}{N^{2}} \sum_{\mathbf{k}, \mathbf{k}^{\prime}} v_{\alpha}(\mathbf{k}) v_{\beta}\left(\mathbf{k}^{\prime}\right) \sum_{\sigma \tau}(-\sigma \tau) G_{\sigma}(\mathbf{k}) G_{\tau}(\mathbf{k}) \\
& \times\left\{1-\left[\Lambda_{\sigma \tau}^{e h} G_{\sigma} G_{\tau}\right] \cdot\right\}^{-1}\left(\mathbf{k}, \mathbf{k} ; \mathbf{k}^{\prime}-\mathbf{k}\right),
\end{aligned}
$$

i.e., the exact solutions for the full vertex $\Gamma$ and the irreducible vertex $\Lambda^{\text {eh }}$ in the two representations (8) and (10) are identical. The vertex $\Lambda^{\text {eh }}$ contains all two-particle scatterings that cannot be disconnected by cutting one electron and one hole propagator and hence includes all multiple scatterings described by the Bethe-Salpeter equations in the electronelectron and vertical channels.

Expression (10) remains positive unless an eigenvalue of the denominator becomes negative. For sufficiently weak disorder it is known that in $d>2$ the eigenvalues are always positive, i.e., $\left\|\Lambda_{\sigma \tau}^{e h} G_{\sigma} G_{\tau}\right\| \lesssim 1$. The conductivity can only become negative if the maximal/minimal eigenvalue of the denominator goes through infinity/zero, respectively. In the former case $(\sigma \rightarrow 0)$ an Anderson transition to the localized phase takes place, and in the latter $(\sigma \rightarrow \infty)$ the system becomes superconducting. For noninteracting disordered systems without electron-phonon interaction the latter transition can be ruled out. In both cases one would have to take the transition into the new phase explicitly into account to guarantee positivity of the conductivity.

We note that only the nonlocal part with odd parity with respect to reflections in $\mathbf{k}$ and $\mathbf{k}^{\prime}$ of the vertex $\Lambda^{\text {eh }}\left(\mathbf{k}, \mathbf{k} ; \mathbf{k}^{\prime}\right.$ -k) contributes to the conductivity. We obtain its leading asymptotic term if we use the representation $\Gamma=\{1$ $\left.-\left[\Lambda^{e h} G G\right] \cdot\right\}^{-1} \Lambda^{e h}$ and solve it for $\Lambda^{e h}$, i.e., $\Lambda^{e h}$ $=\Gamma\{\bullet[G G \Gamma]+1\}^{-1}$. Using the vertex $\Gamma$ from Eq. (7) one finds in the order $O(1 / d)$

$$
\begin{aligned}
\Lambda^{e h}\left(\mathbf{k}_{1}, z_{1}, \mathbf{k}_{2}, z_{2} ; \mathbf{q}\right)= & \Lambda\left(z_{1}, z_{2}\right)+\left(1-\Lambda\left(z_{1}, z_{2}\right) G\left(z_{1}\right)\right. \\
& \left.\times G\left(z_{2}\right)\right)^{2}\left[\Gamma\left(\mathbf{k}_{1}, z_{1}, \mathbf{k}_{2}, z_{2} ; \mathbf{q}\right)\right. \\
& \left.-\Gamma^{e h}\left(\mathbf{k}_{2}-\mathbf{k}_{1} ; z_{1}, z_{2}\right)\right] .
\end{aligned}
$$

The irreducible vertex (11) together with the multiplication scheme (9b) used in Eq. (10) leads to an integral-equation representation of the conductivity. In the limit $d \rightarrow \infty$ the momentum convolutions decouple. This fact helps us to further simplify the expression for the conductivity. To derive the leading asymptotic contribution from the nonlocal part of $\Lambda^{\text {eh }}$ to the conductivity we have to calculate the momentum convolutions on the level of order $O(1 / d)$ so that the velocities appear in squares and the momentum integrals do not vanish.

In the following we consider a hypercubic lattice where only the diagonal (longitudinal) conductivity remains. We expand the denominator in Eq. (10) in powers of $\Lambda^{e h}$ as 


$$
\begin{aligned}
\operatorname{Re} \sigma_{\alpha \alpha}= & \frac{e^{2}}{4 \pi} \sum_{\sigma \tau}(-\sigma \tau) \int d \mathbf{k} d \mathbf{k}^{\prime} v_{\alpha}(\mathbf{k}) G_{\sigma}(\mathbf{k}) G_{\tau}(\mathbf{k}) \\
& \times\left[\delta\left(\mathbf{k}-\mathbf{k}^{\prime}\right)+\sum_{n=0}^{\infty} \int d \mathbf{k}_{1} \ldots d \mathbf{k}_{n}\left\langle\mathbf{k}\left|\Lambda_{\sigma \tau}^{e h}\right| \mathbf{k}_{1}\right\rangle\right. \\
& \times G_{\sigma}\left(\mathbf{k}_{1}\right) G_{\tau}\left(\mathbf{k}_{1}\right) \ldots\left\langle\mathbf{k}_{n}\left|\Lambda_{\sigma \tau}^{e h}\right| \mathbf{k}^{\prime}\right\rangle G_{\sigma}\left(\mathbf{k}^{\prime}\right) \\
& \left.\times G_{\tau}\left(\mathbf{k}^{\prime}\right)\right] v_{\alpha}\left(\mathbf{k}^{\prime}\right),
\end{aligned}
$$

where $\left\langle\mathbf{k}_{l}\left|\Lambda_{\sigma \tau}^{e h}\right| \mathbf{k}_{l+1}\right\rangle=\Lambda_{\sigma \tau}^{e h}\left(\mathbf{k}_{l}, \mathbf{k}_{l} ; \mathbf{k}_{l+1}-\mathbf{k}_{l}\right), l \leqslant n$, and $\mathbf{k}_{0}$ $\equiv \mathbf{k}$ and $\mathbf{k}_{n+1} \equiv \mathbf{k}^{\prime}$. Only odd powers of $\mathbf{k}_{l}, \mathbf{k}_{l+1}$ in $\Lambda_{\sigma \tau}^{e h}\left(\mathbf{k}_{l}, \mathbf{k}_{l} ; \mathbf{k}_{l+1}-\mathbf{k}_{l}\right)$ contribute to the integral. We expand each vertex function $\Lambda^{\text {eh }}$ in Eq. (12) in powers of $v_{\alpha}\left(\mathbf{k}_{l}\right)$ and $v_{\alpha}\left(\mathbf{k}_{l+1}\right)$. In the leading asymptotic order, i.e., including $O(1 / d)$, only linear terms in the velocities remain and the momentum conservation in vertices is relaxed. Hence the expansion coefficients of the vertices are momentum independent and intermediate integrations over momenta decouple. Using these simplifications we arrive at a mean-fieldlike expression for the dc conductivity

$$
\operatorname{Re} \sigma_{\alpha \alpha}=\frac{e^{2}}{4 \pi} \sum_{\sigma \tau}(-\sigma \tau) \frac{\left\langle v_{\alpha}^{2} G_{\sigma} G_{\tau}\right\rangle}{1-\left\langle v_{\alpha}^{2} G_{\sigma} G_{\tau}\right\rangle\left\langle\Lambda_{\sigma \tau}^{\prime \alpha}\right\rangle},
$$

where $\left\langle v_{\alpha}^{2} G_{\sigma} G_{\tau}\right\rangle=N^{-1} \Sigma_{\mathbf{k}} v_{\alpha}(\mathbf{k})^{2} G_{\sigma}(\mathbf{k}) G_{\tau}(\mathbf{k})$ and

$$
\left\langle\Lambda_{\sigma \tau}^{\prime \alpha}\right\rangle=\frac{1}{N^{2}} \sum_{\mathbf{k}, \mathbf{k}^{\prime}} \frac{\delta^{2}}{\delta v_{\alpha}(\mathbf{k}) \delta v_{\alpha}\left(\mathbf{k}^{\prime}\right)} \Lambda_{\sigma \tau}^{e h}\left(\mathbf{k}, \mathbf{k} ; \mathbf{k}^{\prime}-\mathbf{k}\right) .
$$

In the asymptotic limit $d \rightarrow \infty$ the irreducible vertex $\Lambda^{\text {eh }}$ is determined from Eqs. (6), (7), and (11). We note that in this limit one has $\left\langle\Lambda_{\sigma \tau}^{\prime \alpha}\right\rangle \sim O(1)$ and $\left\langle v_{\alpha}^{2} G_{\sigma} G_{\tau}\right\rangle \sim O(1 / d)$. For $\left\langle\Lambda_{\sigma \tau}^{\prime \alpha}\right\rangle=0$ (as, for example, in the case of a k-independent $\left.\Lambda_{\sigma \tau}^{e h}\right)$ the resulting expression for the conductivity reduces to the CPA result

$$
\operatorname{Re} \sigma_{\alpha \alpha}^{\mathrm{CPA}}=\frac{e^{2}}{4 \pi} \sum_{\sigma \tau}(-\sigma \tau)\left\langle v_{\alpha}^{2} G_{\sigma} G_{\tau}\right\rangle .
$$

This is precisely the mean-field conductivity defined from the $d \rightarrow \infty$ limit, with $\operatorname{Re} \sigma_{\alpha \alpha}^{\mathrm{CPA}} \sim O(1 / d)$ due to $v_{\alpha}^{2} \sim O(1 / d)$. The denominator in Eq. (13a) then contains the leading asymptotic contribution from the vertex corrections to the conductivity.

We note that the self-energy $\Sigma$ and the vertex function $\Lambda^{e h}$ are connected via a Ward identity. Velický ${ }^{18}$ showed that the CPA self-energy $\Sigma$, Eq. (2), and the CPA-vertex $\Lambda$, Eq. (4), fulfill the Ward-identity exactly. In our case, where we use the local CPA self-energy and the nonlocal part of the vertex function $\Lambda^{e h}$, Eq. (11), or rather $\left\langle\Lambda_{\sigma \tau}^{\prime \alpha}\right\rangle$ from Eq. (13b), the Ward identity is fulfilled only asymptotically in the leading order of $1 / d$ for both quantities. This is fully consistent with the spirit of the simplification we made in deriving the mean-field expression for the conductivity with vertex corrections (13).

Generally, i.e., in finite dimensions, one has to perform k-integrals in Eq. (13). In this case the conductivity does not have the typical mean-field form where the $\mathbf{k}$-dependence enters only through the dispersion $\epsilon(\mathbf{k})$ such that k-integrals can be replaced by integrals over the density of states. A reduction to such a mean-field expression is possible if we include only the leading contribution in $1 / d$ to $\left\langle\Lambda_{\sigma \tau}^{\prime \alpha}\right\rangle$, i.e., include only the leading vertex corrections in $1 / d$ to the conductivity. To do so, we insert Eq. (11) into Eq. (13b) and make use of the simplified momentum dependence of convolutions of one-particle propagators where the non-local part of two-particle dispersion relation is replaced by the function $X(\mathbf{k})=\left(t^{*} / d\right) \sum_{\nu=1}^{d} \cos \mathbf{k}_{\nu}$. When momenta in high dimensions are summed it behaves as a Gaussian random variable with variance $t^{* 2} / 2 d .{ }^{19}$ On a hypercubic lattice the square of the velocity factorizes and contributes a factor $\left\langle v_{\alpha}^{2}\right\rangle=t^{* 2} / 2 d$. Thus, in leading asymptotic order only local vertices and integrals over the density of states determine the conductivity:

$$
\operatorname{Re} \sigma_{\alpha \alpha}=\left(\frac{e^{2} t^{* 2}}{8 \pi d}\right) \sum_{\sigma \tau} \frac{(-\sigma \tau)\left\langle G_{\sigma} G_{\tau}\right\rangle}{1+\frac{t^{* 2}}{2 d}\left\langle G_{\sigma} G_{\tau}\right\rangle \Lambda_{\sigma \tau}\left(1-\Lambda_{\sigma \tau} G_{\sigma} G_{\tau}\right)\left[\gamma_{\sigma \tau}\left\langle G_{\sigma}^{2}\right\rangle\left\langle G_{\tau}^{2}\right\rangle-\gamma_{\sigma \sigma}\left\langle G_{\sigma}^{2}\right\rangle^{2}-\gamma_{\tau \tau}\left\langle G_{\tau}^{2}\right\rangle^{2}\right]}
$$

where $\left\langle G_{\sigma} G_{\tau}\right\rangle$ is defined as in Eq. (13) and $\gamma_{\sigma \tau}=\gamma\left(E_{F}\right.$ $\left.+i \sigma 0^{+}, E_{F}+i \tau 0^{+}\right)$. We immediately see that expression (15) is exact to $O(1 / d)$ for the conductivity and to $O\left(1 / d^{2}\right)$ concerning the vertex corrections. For the conductivity itself to be exact to $O\left(1 / d^{2}\right)$ an additional contribution in the numerator due to self-energy corrections of $O(1 / d)$ would have to be included. This, however, would not affect the vertex corrections in the leading order. We note once more that results obtained from a direct expansion of the conductivity or the self-energy in powers of $1 / d$ would not be guaranteed to be physical, e.g., the conductivity may become negative and the self-energy non-Herglotz.

Vertex corrections to the conductivity are particularly important in low dimensions $(d=1,2)$, where they lead to Anderson localization even for arbitrarily weak disorder. This is due to the diffusion pole in the two-particle propagator with energies from different complex half-planes. Equation (13), which was derived in the limit of high dimensions, is not expected to be applicable in low dimensions, or to describe Anderson localization. Nevertheless, it is apparent 

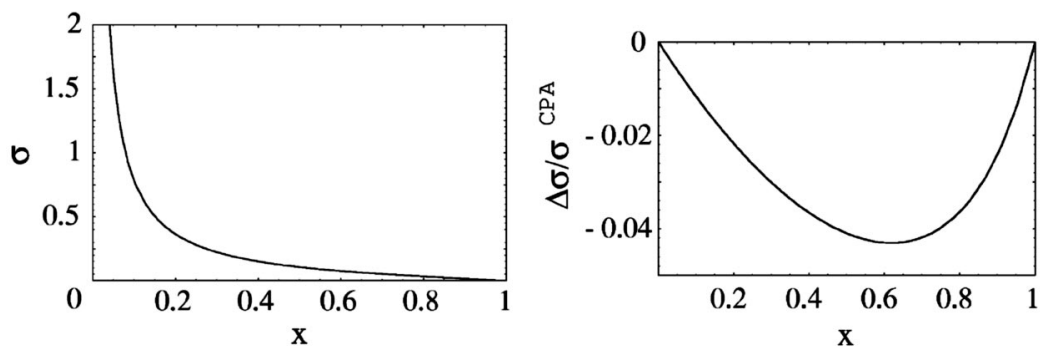

FIG. 3. Mean-field conductivity with vertex corrections $\sigma=\operatorname{Re} \sigma_{\alpha \alpha}^{\text {perc }}$ from Eq. (16a) and relative difference $\Delta \sigma / \sigma^{\mathrm{CPA}}=\operatorname{Re} \sigma_{\alpha \alpha}^{\mathrm{perc}} / \operatorname{Re} \sigma_{\alpha \alpha}^{\mathrm{CPA} \text {,perc }}$ -1 as a function of the concentration $x$ for percolation disorder in $d=3$.

$$
\operatorname{Re} \sigma_{\alpha \alpha}^{\mathrm{CPA}, \mathrm{perc}}=\left(\frac{e^{2}}{4 \pi d}\right) \frac{2(1-x)}{x(2-x)}
$$

diffusion pole (in $\Gamma^{e e}$ ) and thereby also a Cooper pole describing coherent back scattering. For $(-\sigma \tau)=+1$ the k-integrals over the bare Cooper poles in Eq. (13b) diverge in dimensions $d \leqslant 2$. Hence $d=2$ naturally appears as a critical dimension in our high dimensional approximation where the mean-field description must break down.

In dimensions $d>2$ the effect of the diffusion pole in $\Gamma^{e e}$ on the electrical conductivity is not as strong in general and depends on the strength and type of disorder, band filling and the lattice structure. Below we demonstrate this trend with the help of two different disorder distributions. We put the Fermi energy into the band center and choose the dispersion of a $d=\infty$ Bethe lattice, with next-neighbor hopping amplitude $t^{*}=1$ to simplify the relation between the self-energy and the local propagator. First we choose the simplest disorder model, i.e., a percolation-type disorder distribution with $V_{i}=0, \infty$ occurring with probabilities $1-x, x$, respectively. A lattice site with infinite potential is unavailable for the electron motion; the disorder strength is parametrized by the concentration $x$. The conductivity from Eq. (15) can now be evaluated explicitly. The result is

$$
\operatorname{Re} \sigma_{\alpha \alpha}^{\mathrm{perc}}=\left(\frac{e^{2}}{4 \pi d}\right)\left\{\frac{\frac{1-x}{x}}{1+\frac{3}{2 d} \frac{x(1-x)}{(2-x)^{2}}}+\frac{\frac{1-x}{2-x}}{1+\frac{1}{2 d} \frac{x^{2}(1-x)}{(2-x)^{3}}}\right\} .
$$

The conductivity (16a) remains positive everywhere and is a monotonically decreasing function of the disorder strength $x$. Note that in the limit $x \rightarrow 1$ the strength of the disorder becomes effectively infinite since the local irreducible vertex diverges as $\Lambda_{\sigma \tau}=x /(1-x)$. However, in this limit the density of states approaches zero as $\sqrt{1-x}$. Hence $\left\langle G_{\sigma} G_{\tau}\right\rangle=$ $-\sigma \tau(1-x) /[1+\sigma \tau(1-x)]$, so that the denominator of Eq. (15) remains positive and finite as expressed by Eq. (16a). Figure 3 shows that the vertex corrections decrease the oneparticle (i.e., CPA) conductivity for all concentration values $x$.

Another and more important example is the standard binary alloy with two values of the random potential $V_{i}=$ $\pm \Delta$ with equal probability, where we obtain

$$
\begin{aligned}
\operatorname{Re} \sigma_{\alpha \alpha}^{\mathrm{bin}}=\left(\frac{e^{2}}{4 \pi d}\right)\left\{\frac{\frac{1-\Delta^{2}}{\Delta^{2}}}{1-\frac{1}{2 d} \frac{\Delta^{2}\left(1-\Delta^{2}\right)}{\left(2-\Delta^{2}\right)^{2}}}\right. \\
\left.+\frac{\frac{1-\Delta^{2}}{2-\Delta^{2}}}{1+\frac{1}{2 d} \frac{\Delta^{4}\left(1-\Delta^{2}\right)}{\left(1-2 \Delta^{2}\right)^{2}\left(2-\Delta^{2}\right)^{3}}}\right\} .
\end{aligned}
$$

Figure 4 shows that vertex corrections cause the conductivity to slightly increase with respect to the CPA result

$$
\operatorname{Re} \sigma_{\alpha \alpha}^{\mathrm{CPA}, \mathrm{bin}}=\left(\frac{e^{2}}{4 \pi d}\right) \frac{2\left(1-\Delta^{2}\right)}{\Delta^{2}\left(2-\Delta^{2}\right)}
$$

at weak and moderate disorder strength. Around $\Delta=1 / \sqrt{2}$, the two-particle scatterings described by the vertex corrections result in a decrease of the CPA conductivity. For stronger disorder the conductivity (17a) remains positive and is always smaller than the CPA result (17b) up to the split-band limit $\Delta=1$, where it vanishes due to the vanishing of the density of states. Both conductivities (17a) and (17b) monotonically decrease as functions of the disorder strength. However, the largest relative suppression of the CPA conductivity due to vertex corrections occurs at $\Delta=1 / \sqrt{2}$ where the denominator of the second term in Eq. (17a), i.e., the terms with $\sigma \tau=1$ in Eq. (15), diverges and changes sign. Note that if the denominators in Eq. (17a) were expanded only to the
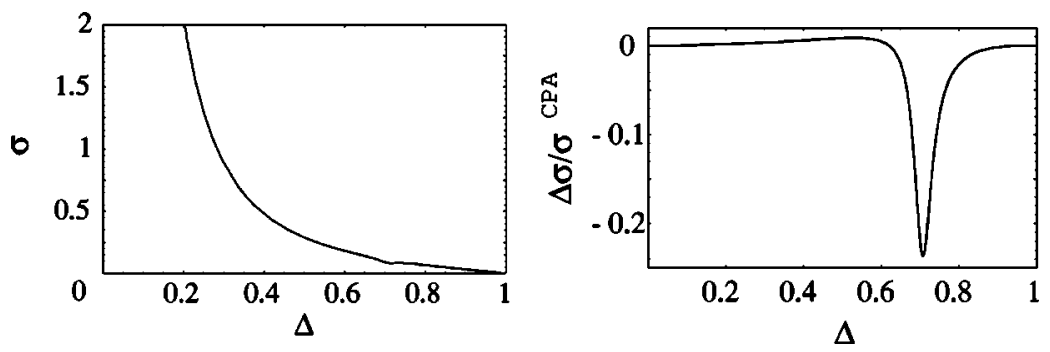

FIG. 4. Mean-field conductivity with vertex corrections $\sigma=\operatorname{Re} \sigma_{\alpha \alpha}^{\text {bin }}$ from Eq. (17a) and relative difference $\Delta \sigma / \sigma^{\mathrm{CPA}}=\operatorname{Re} \sigma_{\alpha \alpha}^{\mathrm{bin}} / \operatorname{Re} \sigma_{\alpha \alpha}^{\mathrm{CPA} \text {,bin }}$ -1 as a function of the disorder strength $\Delta$ for binary alloy in $d=3$. 
leading order in $1 / d$, the conductivity would turn negative and hence become unphysical around $\Delta=1 / \sqrt{2}$. This explicitly demonstrates the importance of calculating the conductivity via approximations to the irreducible vertex $\Lambda^{e h}$, as proposed in this paper, instead of evaluating the conductivity corrections to the single-bubble diagram directly from the full vertex $\Gamma$.

The results for the two models presented above demonstrate that the effect of vertex corrections in high dimensions is in general nonuniversal, i.e., scattering processes from the random potential described by the vertex corrections can decrease or increase the single-bubble conductivity. The effect of vertex corrections then depends on band structure, band filling, and disorder distribution and strength. This situation is very different from low dimensions $(d \leqslant 2)$ where the vertex corrections imply universal behavior since they contain (Cooper) poles which lead to the breakdown of diffusion, and hence to electron localization, irrespective of the disorder distribution and strength. ${ }^{13,14}$

In conclusion, we derived a mean-field expression for the vertex corrections to the electrical dc conductivity which becomes exact in the asymptotic limit of high lattice dimensions. To warrant the conductivity to be non-negative it was expressed as a functional of the irreducible vertex function in the electron-hole channel $\Lambda^{e h}$. We calculated the leading high-dimensional asymptotics of the nonlocal part of $\Lambda^{\text {eh }}$ and thereby derived an expression in closed form for the dc conductivity including vertex corrections. Although this mean-field approximation does not describe Anderson localization, it goes systematically beyond the CPA. The result, Eq. (13), can be used as a mean-field formula for calculating the effects of vertex corrections to the electrical conductivity in, e.g., three-dimensional alloys, and may serve as a starting point for improved approximation schemes beyond the CPA limit. To include the essentials of the physics of Anderson localization one needs to improve the mean-field approximation for the irreducible vertex presented in this paper. A minimal requirement for this is a self-consistent theory for the nonlocal part of the vertex $\Lambda^{\text {eh }}$ which may, for example, be obtained from a parquet approximation. ${ }^{16}$

This work was supported in part by Grant No. 202/98/ 1290 of the Grant Agency of the Czech Republic (V.J.) and by the Sonderforschungsbereich 484 of the Deutsche Forschungsgemeinschaft.
${ }^{1}$ B. Velický, S. Kirkpatrick, and H. Ehrenreich, Phys. Rev. 175, 745 (1968).

${ }^{2}$ R. J. Elliot, J. A. Krumhansl, and P. L. Leath, Rev. Mod. Phys. 46, 465 (1974).

${ }^{3}$ E. Müller-Hartmann, Solid State Commun. 12, 1269 (1973).

${ }^{4}$ F. J. Wegner, Phys. Rev. B 19, 783 (1979).

${ }^{5}$ W. Metzner and D. Vollhardt, Phys. Rev. Lett. 62, 324 (1989).

${ }^{6}$ R. Vlaming and D. Vollhardt, Phys. Rev. B 45, 4637 (1992).

${ }^{7}$ V. Janiš and D. Vollhardt, Phys. Rev. B 46, 15712 (1992).

${ }^{8}$ A. Georges, G. Kotliar, W. Krauth, and M. Rozenberg, Rev. Mod. Phys. 68, 13 (1996).

${ }^{9}$ V. Janiš, Phys. Rev. Lett. 83, 2781 (1999).

${ }^{10}$ M. H. Hettler, M. Mukherjee, M. Jarrell, and H. R. Krishnamurthy, Phys. Rev. B 61, 12739 (2000).

${ }^{11}$ A. Khurana, Phys. Rev. Lett. 64, 1990 (1990).

${ }^{12}$ Note that the definition of a "mean-field conductivity" is ambiguous, since we can implement the high-dimensional asymptotics in two ways. We can either analyze directly the diagrams contributing to the conductivity, find out the leading asymptotic contribution in high dimensions and define the result as a meanfield conductivity. This will lead to the single-bubble diagram of order $1 / d$. Or we may start with the two-particle vertex $\Gamma$, take its leading asymptotic form, insert it into the Kubo formula for the current-current correlation function and identify the resulting expression with a mean-field conductivity. The vertex corrections appearing in this mean-field conductivity cannot be eliminated by resorting to symmetry, (Ref. 9). They are of order $O\left(1 / d^{2}\right)$ and do not change the leading high-dimensional asymptotics of the single-bubble diagram. The difference between the two definitions of the mean-field conductivity becomes important, however, in finite dimensions, where one actually wants to use the high-dimensional asymptotics as a mean-field approximation.

${ }^{13}$ P. A. Lee and R. V. Ramakrishnan, Rev. Mod. Phys. 57, 287 (1985).

${ }^{14}$ D. Vollhardt and P. Wölfle, in Electronic Phase Transitions, edited by W. Hanke and Yu. V. Kopaev (Elsevier, Amsterdam, 1992), p. 1.

${ }^{15}$ M. Jarrell and H. R. Krishnamurthy, cond-mat/0006431.

${ }^{16}$ V. Janiš, cond-mat/0010044.

${ }^{17}$ V. Janiš, Phys. Rev. B 60, 11345 (1999).

${ }^{18}$ B. Velický, Phys. Rev. 184, 614 (1969).

${ }^{19}$ E. Müller-Hartmann, Z. Phys. B: Condens. Matter 74, 507 (1989). 